\title{
Electron Microscopy Advances for Studies of Catalysis at Atomic-Resolution and at Ambient Pressure Levels
}

\author{
C. F. Elkjær ${ }^{1,2}$, S. B. Vendelbo ${ }^{1,3}$, H. Falsig ${ }^{1}$, I. Puspitasari ${ }^{3}$, P. Dona ${ }^{4}$, L. Mele ${ }^{4}$, B. Morana ${ }^{5}$, \\ R. van Rijn ${ }^{6}$, B. J. Nelissen ${ }^{7}$, J. F. Creemer ${ }^{5}$, Ib Chorkendorff ${ }^{2}$, P. J. Kooyman ${ }^{3}$, S. Helveg ${ }^{1}$ \\ 1. Haldor Topsoe A/S, Nymøllevej 55, DK-2800 Kgs. Lyngby, Denmark \\ 2. CINF, Technical University of Denmark, Fysikvej building 307, 2800 Kgs. Lyngby, Denmark \\ 3. ChemE, Delft University of Technology, Julianalaan 136, 2628 BL Delft, The Netherlands \\ 4. FEI Company, Acthtseweg Noord 5, 5651 GG Eindhoven, The Netherlands \\ 5. DIMES-ECTM, Delft University of Technology, P.O. Box 5053, 2600 GB Delft, The Netherlands \\ 6. Leiden Probe Microscopy BV, Niels Bohrweg 2, 2333 CA Leiden, The Netherlands \\ 7. Albemarle Catalyst Company BV, P.O. Box 37650, 1030 BE Amsterdam, The Netherlands
}

The size, shape and surface structure of nanoparticles affect their catalytic properties in ways that are difficult to predict. Because the nanoparticles tend to adapt a geometrical, compositional and electron structure dictated by the actual gaseous environment, it is important to characterize surface structure and dynamics under exposure to reaction conditions as close as possible to technological relevant conditions.

Here we present recent advances of a micro-electro-mechanical system (MEMS) device enabling the first observations of nanoparticles catalyzing gas-phase reactions at ambient pressure levels by simultaneous high-resolution transmission electron microscopy (HRTEM), mass spectrometry (MS) and reaction calorimetry (CAL) [1-3]. The MEMS device contains a narrow gas flow channel extending just $4.5 \mu \mathrm{m}$ along the electron beam direction. Herein, 15-nm-thin electron-transparent windows are placed, enabling HRTEM at ambient pressures. The windows are distributed between windings of a fourelectrode coil that allows heating and temperature-control of the reactor channel (Fig. 1).

The nanoreactor is used for studying Pt nanoparticles (Fig. 1) under the oscillatory oxidation of CO. During exposure to $\mathrm{CO}-\mathrm{O}_{2}-\mathrm{He}$ gas mixtures at 1 bar total pressure, aberration-corrected HRTEM reveals that the nanoparticles undergo reversible and periodic changes in their shape while the MS and CAL data shows synchronous oscillations in global CO conversion (Fig. 2). These non-invasive observations were ensured by conducting HRTEM at low electron dose-rates [3-5]. Specifically, as the reaction rate increases, the nanoparticle shape changes from a rounded towards more facetted shape and subsequently, as the reaction rate decreases, the shape reverts back towards the more rounded shape. Moreover, HRTEM reveals that the more facetted nanoparticles are terminated by extended (111) planes and that the more rounded nanoparticles are terminated by (111) planes and a higher abundance of step sites.

The experimental observations of the oscillatory $\mathrm{CO}$ oxidation reaction are consistently explained by a time-dependent model that incorporates density functional theory and microkinetic modeling of $\mathrm{CO}$ oxidation on the (111) planes and step sites of $\mathrm{Pt}$ as well as a description of convection and diffusion in the gas flow channel. Specifically, the model demonstrates for the first time that a dynamic and reversible refacetting of $\mathrm{Pt}$ nanoparticles represents a mechanism for reaction oscillations. These findings therefore demonstrate that the nanoreactor is a beneficial complement to the multitude of in situ and operando spectroscopic techniques in heterogeneous catalysis and nanoparticle research, because gas-surface phenomena are uncovered at meaningful conditions at the atomic-scale. 
References:

[1] J.F. Creemer et al, Proceedings: IEEE MEMS (2011), p. 1103-1106.

[2] S.B. Vendelbo et al, Ultramicroscopy 133 (2013), p. 72-79

[3] S.B. Vendelbo et al. Nature Materials 13 (2014), p. 884-890.

[4] J.R. Jinschek, S. Helveg, Micron 43 (2012), p. 1156-1168.

[5] S. Helveg, C.F. Kisielowski, J.R. Jinschek, P. Specth, G. Yuan, H. Frei, Micron 68 (2015) 176-185.
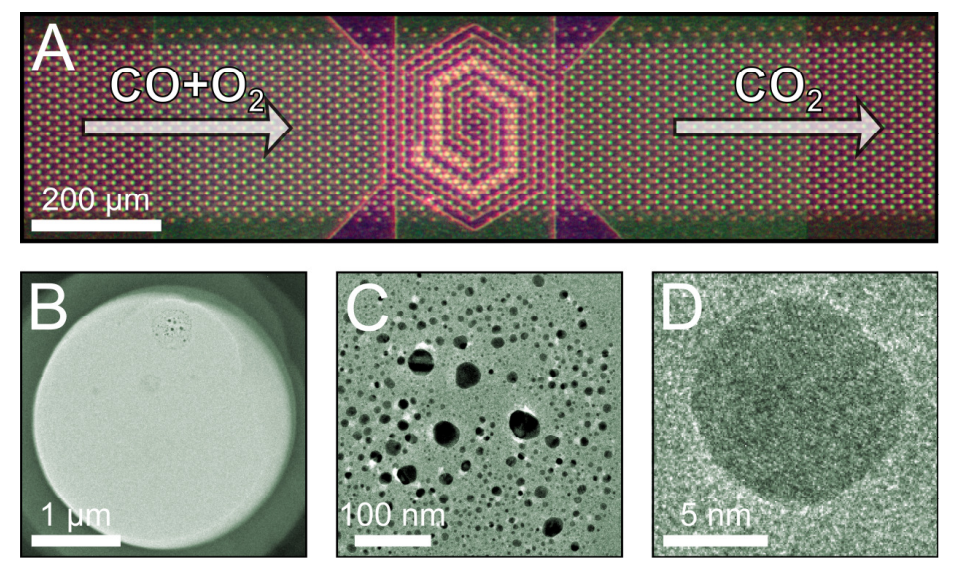

Figure 1. CO oxidation catalyzed by Pt nanoparticles in the nanoreactor. (A) Optical micrograph of the nanoreactor channel with the reaction zone defined by the heater coil and electron-transparent windows for HRTEM. (B-C) Electron micrographs of an electron transparent window and of Pt nanoparticles dispersed on the window. (D) Low dose-rate HRTEM of a Pt nanoparticle in 1 bar CO: $\mathrm{O}_{2}: \mathrm{He}=3: 24: 55$.

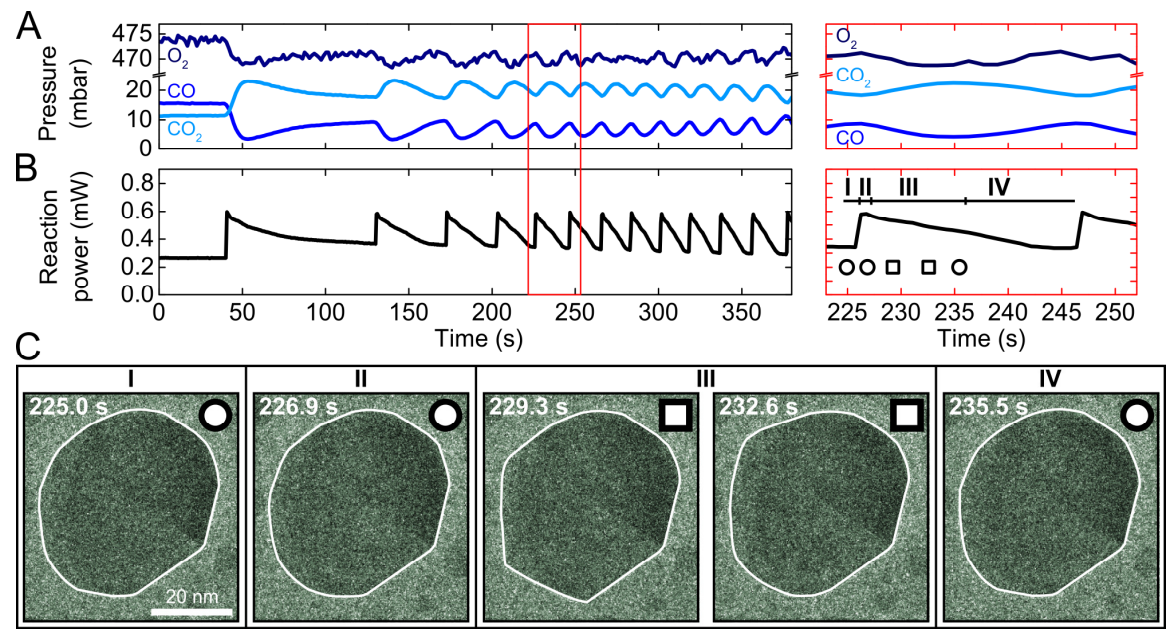

Figure 2. Oscillatory oxidation of $\mathrm{CO}$ catalyzed by Pt nanoparticles in the nanoreactor. Simultaneous, time-resolved (A) mass spectrometry of the $\mathrm{CO}, \mathrm{O}_{2}$ and $\mathrm{CO}_{2}$ pressures, (B) reaction power and $(\mathrm{C})$ HRTEM of a Pt nanoparticle. Reaction conditions: 1.0 bar of $\mathrm{CO}: \mathrm{O}_{2}: \mathrm{He}$ at 3\%:42\%:55\% and temperature is $659 \mathrm{~K}$. Adapted from [3]. 